\title{
DYSENTERY AND COLITIS
}

\author{
By Sir Philip Manson-Bahr, C.M.G., D.S.O., M.D., F.R.C.P.
}

This is an ever-recurrent subject of considerable contemporary importance and it is one which, in view of therapeutic advances, calls for a periodic review. As a result of the recent war large numbers of ex-Service personnel who have served in India, Burma and in the Far East are still suffering from and will continue to exhibit recurring symptoms of these disorders for some time to come.

Of the bacillary dysenteries there is fortunately little to be said. In the acute dysenteries of bacillary origin a great therapeutic victory has been won by the introduction of the special sulphonamides-sulphaguanidine and sulphasuccidine. Not only has this disease been robbed of its terrors, but its sequelae, which loomed so large some 25-30 years ago, have almost completely disappeared. No longer do we encounter those alterations in the lumen of the large intestine, those peritoneal adhesions and that peculiarly intractable ulceration formerly known as chronic bacillary dysentery. Even the granular inflammation of the lower rectum, or 'granular proctitis,' the unhealed remnant of bacillary necrosis of the mucosa, is rarely seen nowadays.

These sulphonamides have amply proved their worth, of that there can be no question. And probably also they are not only of curative, but also of prophylactic value, because it appears to have been established that by taking sulphaguanidine in daily gramme doses the onset of these dysenteries may be effectually prevented. It is due to these measures that the incidence of bacillary dysentery in the Eastern theatres of war sank, during 1945, to insignificant proportions and that the mortality rate declined to less than $I$ in 5,000 . These are indeed remarkable figures.

Though sulphaguanidine and sulphasuccidine are especially valuable in the more toxic bacillary infections caused by Shiga and
Flexner bacilli, they appear to be less effective in Sonne dysentery, which is so prevalent in this country at present. There is, however, some evidence that the more recently introduced compound, phthalystatin, possesses greater bacteriostatic powers in this infection. However, there is some comfort in the contemplation that Sonne dysentery produces considerably less damage to the bowel surface than the other organisms of this group.

Whether exposure on a large scale to these specific infections gives rise to a much greater incidence of so-called idiopathic ulcerative colitis amongst men of military age, which has been recently so much in evidence, remains a moot point. It is undoubtedly true that in wartime the numbers of a very acute form of ulcerative colitis appear to be increased. I would like to stress the great importance at arriving quickly and decisively at a correct diagnosis of this fell and insidious disease. The natural tendency is to regard it in the first instance, when it arises in those areas where the other well-ascertained forms of dysentery are common, as necessarily a manifestation of one of them. I think this especially applies to amoebic dysentery, but certain it is that, if ulcerative colitis is treated continuously and successively and mistakenly for amoebic dysentery with irritating antiamoebic drugs, such as emetine and emetine bismuth iodide, its manifestations are exacerbated.

Manifestly amidst such a whirlpool of fluxes and dysenteries which presents itself in wartime hospitals the onset of idiopathic ulcerative colitis is not readily recognized. Usually little assistance is forthcoming from - the laboratory and I am convinced that the most ready method is by sigmoidoscopy, or even by proctoscopy. By these means the peculiar plush-like appearance of the mucosa can be recognized and its friability and vulnerability 
ascertained. Nor am I convinced that we are yet in possession of any really effective therapeutic measure in combating its ravages. In these acute military cases with extreme emaciation and dehydration a decision must be taken early whether operative measures are justifiable or not. Ileostomy may be, and often is, the only practical method of saving the patient's life, but this must be undertaken before the entire mucosa of the large intestine has been destroyed or the patient overwhelmed by toxaemia.

In less acute cases, or when only limited areas of the bowel are involved-regional ulcerative colitis-then instillation of sulphasuccidine by rectal retention enemata, in 6-8 gm. doses suspended in water and mucilage does, for a time at least, suspend the ulcerative process and leads to lessening of the toxaemia.

Amoebic dysentery is undoubtedly of great importance. The already considerable literature has been enriched by quite a spate of contributions on this disease in the current medical press as a weekly scrutiny of the British Medical Fournal and Lancet amply demonstrates. A great deal of it traverses already familiar ground, though many of our therapeutic heliefs have been sadly shaken.

A fundamental distinction must be made between 'amoebic dysentery' and 'amoebiasis.' The former is associated with ' dysentery': the passage of blood and mucus in the stools, with diarrhoea, colic and abdominal pain. The latter is entirely asymptomatic. The stools may be normal or even constipated, and in them the cysts of Entamoeba histolytica are discovered by routine examination. I am convinced that these two conditions are pathologically and therapeutically quite distinct. In the former the trophozoites of $E$. histolytica are embedded in the bowel wall and have seriously affected the mucosa by the cytolysins they secrete, whilst in the latter the organism plies a coprozoic existence. In the first instance the human host is ill; it may be severely so. In the second he is perfectly well and is not incommoded.

The distinction has been rendered clearer by the introduction of a new quinoxyl compound -diiodoquin - which contains $6_{3}$ per cent. of iodine. This drug is easily tolerated and can be given as an outpatient treatment. A dose of eight tablets daily for $I_{5}$ days suffices to eradicate the infection (i.e. cysts of $E$. histolytica), whilst in the actual disease-amoebic dysentery-it appears to exert little beneficial effect.

Amoebic dysentery may then appear in many disguises. It may, it is true, be severe and the resulting passage of bloodstained stools may result in emaciation and severe anaemia. More often it is chronic. Then the patient suffers mostly from periodic attacks of diarrhoea, abdominal discomfort and sometimes deep-seated pain confined to the caecum or sigmoid colon. In my experience acute abdominal crises, especially when limited to the upper abdomen, are never of amoebic origin.

The diagnosis of amoebic dysentery presents many pitfalls. First, the organisms may be present in large numbers in the faeces in the active vegetative state, especially when quantities of blood and mucus are being evacuated. These motile trophozoites are encountered, only in the active acute stage, whilst the precystic and cystic forms are seen in the chronic stage when the evacuations are faeculent. A great drawback to acute diagnosis and a puzzle to pathologists lies in the fact that the cysts appear in the faeces in a most vicarious fashion. It so happens that on one occasion they may swarm in myriads, whilst in the next they may be absent altogether. It does not appear that the various concentration methods which have been devised materially help in clinching the diagnosis. So it comes about that the clinician has to concert various examinations, clinical, pathological, instrumental and, occasionally, radiographical, in order to obtain a correct diagnosis. I have become increasingly impressed with the value of proctoscopy. By the judicious employment of this procedure amoebae may often be demonstrated in cases in which the faeces have been examined, often on many occasions, in vain. By proctoscopy, which is so easily performed, a good view of the rectal mucosa may be obtained and microscopic preparations can be obtained from scrapings of the diseased surface and the microscopic examination of the exudate immediately carried out. On many occasions this simple method is successful in 
demonstrating the living and active $E$. histolytica. To perform proctoscopy, a simple tubular proctoscope, with obturator, some 6 in. in length is used. No preliminary preparation of the patient is necessary. I find that it is better not to employ a fixed illuminant, such as are fitted into the standard instruments, but an electric bulb introduced within the proctoscope so as to illuminate a limited area of the rectal mucosa. Scrapings can then be made by means of a long-handled Volkmann spoon of any particular lesion encountered. The sample of mucus, blood and mucosa must be $t_{\perp}$ ansferred direct from the bowl of the spoon directly onto a microscope slide, the coverslip must be pressed down and no diluent, such as saline, employed. The microscopic examination must be immediate, any delay is detrimental. Therefore it is preferable to have the microscope on a table in the examination room. If $E$. histolytica is present, the trophozoites can be seen actively progressing between the mucosal cells. I have become increasingly impressed with the varying appearances of the mucosa attacked by $E$. histolytica. This arises from the conviction that the popular idea of the characteristic lesions in the bowel are incorrect. The living pathology differs considerably from the morbid appearances. The earliest lesions consist of small circumscribed hyperaemic areas, later there are small pinhead haemorrhagic depressions which bleed when they are swabbed with cotton wool. The most confusing appearance is in the acute stage when the mucous membrane is so inflamed as to resemble the plush-like haemorrhagic stage of ulcerative colitis. Indeed it is quite intelligible why so many misdiagnoses are arrived at on this very .count. Under these circumstances, I have learned that it is futile to contuse the mucosa by scraping with a sharp instrument to demonstrate the amoebae. The surface of the mucosa should be swabbed with a tightly packed cotton-wool swab, a pledglet of blood and mucus removed, and immediately examined. Sometimes the amoebae may be so numerous as to resemble a solid mosaic of motile trophozoites. Again the mucosa may present a granular appearance; the surface is friable as in the chronic stage of bacillary dysentery. Under the present conditions actual ulceration of the mucosa, such as is depicted in most textbooks, is an extremely rare event. In chronic amoebic dysentery, when $E$. histolytica cysts are numerous in the faeces, the crenation and folding of the mucosa is a prominent feature, whilst the surface is pitted with innumerable depressions like that produced by minute raindrops, giving rise to the classical so-called 'pigskin appearance.' In this stage it is not possible to demonstrate the trophozoites of $E$. histolytica in scrapings, though sometimes precystic forms and cysts may be demonstrated on the mucosal surface. The only reliable clinical sign, apart from the deep tenderness which has already been referred to, is the thickening and induration of the sigmoid colon, but even this may be absent when the amoebic lesions are confined, as they so often are, to the rectum. Amoebic typhlitis is another matter. Here the deepseated pain is restricted to the caecum. This thickened and tender viscus can be palpated in the right iliac fossa and it is in this condition that radiography may be of distinct advantage. In amoebiasis of the colon it is usually particularly unhelpful. The amoebic caecum (by barium enema) gives a distinctive appearance. There are filling defects and, on emptying, a spasm is apparent producing a contracted cone-shaped mass. It is necessary to draw attention to this fact as, in the absence of direct evidence of amoebic infection by faeces examination, it is particularly difficult to diagnose and to differentiate from tubercular disease. We now come to the vexed and much debated question of treatment.

A great deal has been heard and written of the importance of secondary bacterial infections. Whilst not denying that these do actually occur and did play a part in many particularly virulent cases invalided from Burma, I do submit that they do not play any essential part at the present time. Therefore it is inadvisable to submit the average case of intestinal amoebiasis to intensive courses of penicillin and sulphasuccidine therapy before specific antiamoebic treatment is instituted. In the acute phases emetine injections of hypodermic emetine, at the commencement of treatment are, I am convinced, beneficial. After which the routine treatment with emetine and quinoxyl retention enemata should be carried out on the approved lines. The fault is the 
present treatment, which has been subjected to criticism, lies in the quality and preparation of the emetine bismuth iodide. It has recently been shown that it is the dispersibility of the E.B.I. which is the essential factor and the constitution of the covering of the E.B.I. tablet. At present Burroughs Wellcome are putting out a new preparation which is sugarcoated and which readily dissolves in the succus entericus. Some of the older gelatine capsule preparations had the effect of crystallizing the contained E.B.I. which then did not readily disperse. This new preparation is particularly well-tolerated and can be given in gr. iii doses, for a total course of $30 \mathrm{gr}$. I freely admit that relapses still occur, but many of these can be cured by a second course. There is one factor to which, I think, too little attention has been paid. When it has been ascertained by sigmoidoscopy that the mucosa is covered by a dense membrane of muco-pus and detritus then the bowel should be cleansed by high colonic lavage with boracic acid ( 3 i to the pint) or by $2 \frac{1}{2}$ per cent. sodium bicarbonate solution, before the E.B.I. course is instituted. It stands to reason that, unless the bowel surface is reasonably clear, the E.B.I. cannot come into intimate contact with the diseased mucosa. In the chronic stage of the disease diiodoquin is of distinct benefit and appears to have a direct action on the cysts. It has been shown that 120 tablets of this drug suffices to clear up the cyst-passer condition.

Tropical sprue is another form of chronic diarrhoea which has been much in evidence in India and Burma. It has for the first time, in the long history of this disease, appeared in epidemic form and has behaved like an infective disease. Large numbers of men were stricken down at the same time and the disease progressed with hitherto undescribed rapidity. The early stages were quite unlike the generally accepted picture. Steatorrhoea did not appear till the dehydration and emaciation were well advarced. It therefore now appears more likely than ever that sprue is primarily due to a specific infection of the mucosa with special preference for the lower ileum. The later and more familiar phases are probably expressions of vitamin deficiencies brought about by the primary lesions.

There is another characteristic of sprue which appears to me to be almost inexplicable on any aetiological basis. Tropical sprue may arise de novo and run its typical course, with glossitis, steatorrhoea, emaciation, meteorism and megalocytic anaemia, 15 to 20 years after exposure to infection. When this occurs it is certain to produce very real difficulties in diagnosis. Phantasies of carcinoma of the stomach, Addison's disease, anor exia nervosa, chronic cholecystitis or pernicious anaemia may be conjured up. Then, of course, there is the tendency to classify tropical sprue as idiopathic steatorrhoea, pancreatitis, tubercular enteritis, or other forms of fatty diarrhoea. The real distinction lies in this difference. In the vast majority of cases tropical sprue yields readily to treatment. $U p$ to the present the parenteral injection of crude liver extract has held the field. I arge doses are necessary, 4 c.c. or even more, intramuscularly three times a week. Rest in bed and careful dieting are essential. Now at last it appears that a truly specific treatment is in sight as the outcomc of Spies' work on folic acid. Small doses such as $10 \mathrm{mgm}$. twice daily for a period of 5-7 days appears sufficient. Unfortunately in this country folic acid (fovite) is very difficult to obtain. The exact part that folic acid plays in the cure of sprue is not known. That it has some action in the absorption of fats, possibly in the promotion of 'phosphorylation,' appears probable. What is known, and which is the most important, it stops the diarrhoea, reduces the size of the stools, heals the glossitis, increases the appetite and promotes haemopoiesis. The increase of weight is phenomeral. It therefore appears probable that the sure cure of this age-old malevolent and disagreeable disease may now be in sight. 